\title{
Surgical treatments for squamous cell carcinoma of the esophagus reaching to the muscularis mucosa or the upper third of the submucosal layer
}

\author{
JUNYA OGUMA $^{1}$, SOJI OZAWA ${ }^{2}$, YOSHIRO SAIKAWA ${ }^{1}$ and YUKO KITAGAWA $^{1}$ \\ ${ }^{1}$ Department of Surgery, School of Medicine, Keio University, Tokyo 160-8582; ${ }^{2}$ Department of \\ Gastroenterological Surgery, Tokai University School of Medicine, Kanagawa 259-1193, Japan
}

Received December 22, 2009; Accepted March 19, 2010

DOI: 10.3892/ol_00000092

\begin{abstract}
We studied the surgical treatments of patients with either esophageal cancer reaching to the muscularis mucosae (m3) or with a slight invasion of the submucosa (sm1). We reviewed the records and examined the clinicopathological features of 29 patients with $\mathrm{m} 3$ or sm1 esophageal squamous cell carcinoma who had undergone surgery. Lymph node metastasis was noted in $6.3 \%$ (1 patient) of patients with $\mathrm{m} 3$ cancers and in $38.5 \%$ (5 patients) of those with sm1 cancers. The incidence of lymph node metastasis was higher in the sm1 than in the $\mathrm{m} 3$ group, but the difference was not significant. Lymphatic invasion (ly) was noted in $12.3 \%$ (2 patients) of patients with $\mathrm{m} 3$ cancers and in $53.8 \%$ (7 patients) of those with sm1 cancers. The incidence of ly was significantly higher in the $\mathrm{sm} 1$ group than in the $\mathrm{m} 3$ group $(\mathrm{P}<0.05)$. In a multivariate analysis of factors for predicting lymph node metastasis, the presence of ly was the only significant predictor $(\mathrm{P}<0.05)$. The preoperative diagnostic accuracies of endoscopic ultrasonography (EUS), esophagogastroduodenoscopy (EGD) and an upper gastrointestinal series (UGS) for predicting depth of invasion were $27.8,31.0$ and $41.4 \%$, respectively, with the majority of the misdiagnoses being overestimations. In conclusion, we suggested that ly is associated with lymph node metastasis in $\mathrm{m} 3$ or sm1 esophageal cancer. This association is significant for treatment-related decision making.
\end{abstract}

\section{Introduction}

Recent diagnostic developments have led to the detection of superficial esophageal cancer. Consequently, an increase in the number of candidates for endoscopic mucosal resection

Correspondence to: Professor Soji Ozawa, Department of Gastroenterological Surgery, Tokai University School of Medicine, 143 Shimokasuya, Isehara, Kanagawa 259-1193, Japan

E-mail: sozawa@tokai.ac.jp

Key words: esophageal cancer, muscularis mucosa, submucosal layer
(EMR) and endoscopic submucosal dissection (ESD) has been noted. Esophageal cancers extending to the intraepithelium (m1) or lamina propria (m2), in particular, rarely have lymph node metastasis or lymphatic invasion (ly) and are thus curative by local treatment and suitable for treatment with EMR/ ESD $(1,2)$. On the other hand, the frequency of lymph node metastasis from cancer of the muscularis mucosa (m3) or the upper third of the submucosal layer (sm1) is 18.0 and $53.1 \%$, respectively (3). In certain cases local treatment alone would be adequate; however, in other cases treatment for lymph node metastasis would need to be considered. Treatment strategies for $\mathrm{m} 3$ and $\mathrm{sm} 1$ cancers remain controversial. To provide appropriate treatment for these types of cancer, it would be useful to be able to identify patients that need treatment for lymph node metastasis. Diagnosis of lymph node metastasis from esophageal cancer by computed tomography (CT), ultrasonography and FDG-PET currently lacks accuracy. Subsequently, the accurate diagnosis of esophageal cancer prior to treatment presents a challenge. Depth of superficial esophageal cancer is normally diagnosed using endoscopy, endoscopic ultrasonography and a gastrointestinal series. However, despite the availability of these current modalities, accurate diagnosis of tumor depth remains difficult. If a clear diagnosis prior to treatment for $\mathrm{m} 3$ and $\mathrm{sm} 1$ esophageal cancer and accurate predictive factors of lymph node metastasis were to be obtained, it would be possible to accurately identify patients requiring treatment for lymph node metastasis. Thus, more optimal treatment for $\mathrm{m} 3$ and $\mathrm{sm} 1$ esophageal cancer would be possible. Finding solutions to the above-mentioned problems involves a detailed review of the clinicopathological features and outcomes of many cases. However, since m3 and sm1 cancers are relatively uncommon, analyzing the characteristics of a sufficient number of these cases has been difficult. Nevertheless, there has been an increase of studies on the treatment of $\mathrm{m} 3$ and sm 1 cancers along with the increasing incidence at which these lesions are being detected.

The present study reported on the clinicopathological characteristics of patients with $\mathrm{m} 3$ and sm1 cancers that underwent radical surgery in our hospital and whose excisional specimens were examined pathologically. Particular attention was given to lymph node metastasis and ly. To obtain an accurate preoperative diagnosis, preoperative endoscopic 
Table I. Clinicopathological findings of $\mathrm{m} 3 / \mathrm{sm} 1$ esophageal squamous cell carcinoma.

\begin{tabular}{lccr}
\hline & $\mathrm{m} 3(\mathrm{n}=16)$ & $\mathrm{sm} 1(\mathrm{n}=13)$ & P-value \\
\hline Median age (range) & $58(44-80)$ & $58(41-74)$ & 0.607 \\
Lymph node metastasis (\%) & $1(6.3)$ & $5(38.5)$ & 0.095 \\
Lymphatic invasion (\%) & $2(12.5)$ & $7(53.8)$ & 0.047 \\
INFa (\%) & $6(37.5)$ & $2(15.4)$ & 0.364 \\
INFb (\%) & $10(62.5)$ & $11(84.6)$ & 0.858 \\
Size of tumor (mean) & 3.56 & 3.72 & \\
\hline
\end{tabular}

INF, infiltrative growth pattern; INFa (expansive type), expansive growth of tumor nests with a well-demarcated border from surrounding tissue; INFb (intermediate type), intermediate growth pattern, between INFa and c; INFc (infiltrative type), infiltrative growth of tumor nests with an ill-defined border from surrounding tissue.

Table II. Univariate analysis of relative factors for lymph node metastasis.

\begin{tabular}{lccc}
\hline Clinicopathological factors & $\mathrm{n}(+)$ & $\mathrm{n}(-)$ & P-value \\
\hline Age (median; year) & 54 & 61 & 0.12 \\
Gender (M:F) & $3: 3$ & $21: 2$ & 0.09 \\
Size of tumor (mean; cm) & 3.53 & 3.66 & 0.91 \\
Depth (m3:sm1) & $1: 5$ & $15: 8$ & 0.03 \\
ly (+:-) & $5: 1$ & $4: 19$ & 0.01 \\
INF (a:b) & $0: 6$ & $8: 15$ & 0.24 \\
Differentiation (well:mod:poor) & $1: 4: 1$ & $6: 17: 0$ & 0.78 \\
\hline
\end{tabular}

ly, lymphatic invasion.

ultrasonography (EUS), esophagogastroduodenoscopy (EGD), and an upper gastrointestinal series (UGS) were performed. The most appropriate treatment strategy for $\mathrm{m} 3$ and $\mathrm{sm} 1$ esophageal cancers was also examined.

\section{Materials and methods}

The study evaluated the clinicopathological features of 29 patients (m3, 16 patients; sm1, 13 patients) with $\mathrm{m} 3$ or sm1 esophageal squamous cell carcinomas. The patients had undergone radical surgery and excisional specimens had been examined pathologically. In particular, the preoperative diagnostic accuracy of the ability of EUS, EGD and UGS to predict depth of invasion was investigated. The presence of concurrent cancers in other organs as part of the background characteristics of each patient were also examined. Statistical analyses were performed using Student's t-test and the Chi-square test for univariate analysis, and the logistic regression analysis for multivariate analysis. Significant differences were defined as $\mathrm{P}<0.05$.

\section{Results}

Patient characteristics. The median patient age was 58 years (range 41-80). Patients included 25 males and 4 females. Locations of the primary tumors were: 1 in the cervical esophagus (4), 3 in the upper thoracic esophagus (4), 11 in the middle thoracic esophagus (4), 13 in the lower thoracic esophagus (4) and 1 in the abdominal esophagus (4).

Relationship between the muscularis mucosae group and the submucosa group. Table I shows the clinicopathological backgrounds of the patients. Lymph node metastasis was noted in $6.3 \%$ (1 patient) of patients with $\mathrm{m} 3$ cancers and in $38.5 \%$ (5 patients) of those with $\mathrm{sm} 1$ cancers. The incidence of lymph node metastasis was higher in the sm1 group than in the $\mathrm{m} 3$ group, but the difference was not significant (data not shown). The individual case with lymph node metastasis in the $\mathrm{m} 3$ group had a poorly differentiated squamous cell carcinoma with ly. Overall, ly was noted in $12.5 \%$ (2 patients) of patients with $\mathrm{m} 3$ cancers and in $53.8 \%$ ( 7 patients) of those with $\mathrm{sm} 1$ cancers. The incidence of ly was significantly higher in the sm1 group than in the $\mathrm{m} 3$ group. The mean number of metastatic lymph nodes was 1.5 .

Univariate and multivariate analysis of lymph node metastasis. We initially performed a univariate analysis to identify factors with any relationship to lymph node metastasis (Table II). A multivariate logistic regression analysis was then performed using lymph node metastasis as the objective parameter and the depth of invasion and ly, which are relative factors in the univariate analysis, as explanatory parameters. 
Table III. Multivariate analysis of relative factors for lymph node metastasis.

\begin{tabular}{lccl}
\hline & Odds ratio & P-value & \multicolumn{1}{c}{$95 \% \mathrm{CI}$} \\
\hline Depth invasion & 0.26 & 0.2959 & $0.019-3.534$ \\
ly & 15.00 & 0.0181 & $1.219-184.821$ \\
\hline
\end{tabular}

Consequently, ly was the only parameter that was associated with lymph node metastasis (Table III).

Diagnosis before treatment. The preoperative diagnostic accuracies of EUS, EGD and UGS for predicting depth of invasion were $27.8,31.0$ and $41.4 \%$, respectively. In certain cases the depth of invasion was misdiagnosed (0, 3.4 and 3.4\% for each modality, respectively). However, the majority of the misdiagnoses were overestimations $(72.2,65.6$ and $51.7 \%$ for each modality, respectively) (Table IV).

Postoperative course. The median follow-up period was 58 months (7-131 months) for our cases. No local recurrences or death were observed during the follow-up periods.

\section{Discussion}

The most important aspect in deciding the management of $\mathrm{m} 3$ and $\mathrm{sm} 1$ esophageal cancers is lymph node metastasis (5-7). Although CT, ultrasonography, EUS and FDG-PET have been used to diagnose lymph node metastasis in patients with esophageal cancer (8-10), the diagnostic capacity of each of these modalities is limited and the accurate diagnosis of lymph node metastasis prior to treatment is difficult (5). Therefore, when the imaging findings show that esophageal cancer is not accompanied by lymph node metastasis, we usually predict the possibility of lymph node metastasis from its depth of invasion, and treatment is determined accordingly. Understanding the rate of lymph node metastasis associated with varying depth of invasion presents a problem. Consequently, patients with $\mathrm{m} 3$ or sm1 esophageal cancer should be carefully evaluated to determine the most appropriate treatment.

In this study, the univariate analysis suggested that depth of invasion and ly were factors that associated significantly to lymph node metastasis. Additionally, in the multivariate analysis, the presence of ly was a more relative factor than depth of invasion. Therefore, ly is potentially the most effective clinicopathological factor related to lymph node metastasis. Lymphatic invasion is an indicator that shows the transition of cancer cells into the lymphatic flow. In theory, if lymphatic invasion is positive, lymph node metastasis would also appear to be positive. However, there are cases in which lymph node metastasis is not associated with ly and vice versa. The correlation between ly and lymph node metastasis has been shown in gastric (11) and colorectal cancer (12). This correlation is a general relationship irrespective of the depth of invasion (3). Studies have reported on risk factors associated with lymph node metastasis in $\mathrm{m} 3$ or sm1 esophageal cancer. One such study suggested tumors that have ly, larger superficial size and wider muscularis mucosae invasion were associated with a high risk for lymph node metas-
Table IV. Accuracy and tendency of preoperative diagnosis by endoscopicultrasonography, esophagogastroduodenoscopy and upper gastrointestinal series.

Accuracy Underestimated Overestimated
$(\%)$
$(\%)$
(\%)

\section{EUS}

\begin{tabular}{llrl} 
m3 $(\mathrm{n}=9)$ & 33.3 & 0.0 & 66.7 \\
$\mathrm{sm} 1(\mathrm{n}=9)$ & 22.2 & 0.0 & 77.8 \\
Total $(\mathrm{n}=18)$ & 27.8 & 0.0 & 72.2 \\
EGD & & & \\
m3 $(\mathrm{n}=16)$ & 31.3 & 0.0 & 68.7 \\
$\mathrm{sm} 1(\mathrm{n}=13)$ & 30.7 & 7.7 & 61.5 \\
Total $(\mathrm{n}=29)$ & 31.0 & 3.4 & 65.6 \\
UGS & & & \\
m3 $(\mathrm{n}=16)$ & 43.8 & 0.0 & 56.2 \\
sm1 $(\mathrm{n}=13)$ & 38.5 & 15.4 & 46.1 \\
Total $(\mathrm{n}=29)$ & 41.4 & 3.4 & 51.7 \\
\hline
\end{tabular}

EUS, endoscopic ultrasonography; EGD, esophagogastroduodenoscopy; UGS, upper gastrointestinal series.

tasis in patients with $\mathrm{m} 3$ or sm1 esophageal cancer. Moreover, endoscopic mucosal resection (EMR) may be indicated for the treatment of patients with $\mathrm{m} 3$ or sm1 cancer without these characteristics (13). Another study showed that additional treatment, such as radiotherapy, was necessary for patients with tumors that were pathologically diagnosed as being $\mathrm{m} 3$ cancer with ly or sm1 cancer after EMR $(3,14)$. Using a multivariate analysis Tajima et al showed, in the almost same manner as in our study, that the risk factor of lymph node metastasis in esophageal cancer was only ly (6).

In the present study, the preoperative diagnostic accuracies of EUS, EGD and UGS for predicting depth of invasion were $27.8,31.0$ and $41.4 \%$, respectively. Depth of invasion of $\mathrm{m} 3$ or sm1 lesions is difficult to determine accurately in patients with superficial esophageal cancer. Diagnostic criteria for $\mathrm{m} 3$ or sm1 esophageal cancer per examination therefore need to be determined. In EUS, if irregularity or destruction is noted at the third layer from a total of seven layers of the esophageal wall, the tumor is regarded as deeper than $\mathrm{m} 3$ cancer. In endoscopy, $\mathrm{m} 3$ cancer shows slightly larger granules on the surface and sml cancer shows a depressed surface, demonstrating some irregularity and variability in granule size. In UGS, if the lateral view of the depressed or flat tumor is detected as focal straightening, the tumor is regarded as m3 or sm1 cancer. Previous studies showed the diagnostic accuracies of EUS, EGD and UGS for predicting depth of invasion to be as high as $79.6 \%$ (15), $80.2 \%$ (16) and $90 \%$ (17), respectively. In these studies, specialists in each method used the most up-to-date equipment; thus, results obtained were superior to those obtained in this study. Therefore, more accurate investigations should be conducted. Despite the use of the available diagnostic modalities, depth of invasion is overestimated. Depth of invasion is normally underestimated in cases in which the invasion of the submucosal layer by the 
Table V. Clinical outcome of EMR, surgery and radiation for m3/sm1 esophageal squamous cell carcinoma.

\begin{tabular}{|c|c|c|c|c|c|c|}
\hline & \multicolumn{3}{|c|}{ Efficiency of the treatment } & \multicolumn{2}{|c|}{ Safety } & \multirow{3}{*}{$\begin{array}{l}\text { Preservation of } \\
\text { the esophagus }\end{array}$} \\
\hline & \multirow{2}{*}{$\begin{array}{l}5 \text {-year rate of } \\
\text { survival }(\%)\end{array}$} & \multicolumn{2}{|c|}{ Recurrence $(\%)$} & \multirow[t]{2}{*}{ Complication (\%) } & \multirow[t]{2}{*}{ Mortality (\%) } & \\
\hline & & Local & Distant & & & \\
\hline EMR & 77.4 & 8.2 & 6.8 & 21.6 & 0.0 & + \\
\hline Surgery & 84.5 & - & 4.3 & 55.0 & 3.0 & - \\
\hline $\begin{array}{l}\text { Radiation } \\
\text { (T1N0M0) }\end{array}$ & 58.7 & 17.6 & 2.9 & $17.6^{\mathrm{a}}$ & 0.0 & + \\
\hline
\end{tabular}

${ }^{a}>$ NCI-CTC grade III; EMR, endoscopic mucosal resection.

cancer is minor; however, it is overestimated in cases with fibrosis. The use of magnifying endoscopy for evaluating microvessels in the mucosal layer of the lesion was previously reported and appears to be useful for diagnosing the depth of invasion of superficial esophageal cancer $(18,19)$. Investigations as to how accuracy at which the depth of invasion can be diagnosed should therefore be conducted.

Treatment selection for $\mathrm{m} 3$ or $\mathrm{sm} 1$ esophageal cancer include EMR/ESD, surgery and chemoradiotherapy. A search was conducted on PubMed for treatments using the keywords: superficial (or early) esophageal cancer, EMR, surgery and radiation for the period 1998-2008, and the results are shown in Table V (20-22). No report discussed the long-term outcome of chemoradiation; thus Table $\mathrm{V}$ includes findings on only radiation. Regarding clinical outcome, although treatments cannot be compared due to the differences of each background from which these data evolved, the 5-year overall survival rate is high and the recurrence rate is low following surgery. Although local recurrences were noted in EMR and radiation, radiation had a higher recurrence than EMR. With regard to additional treatment for cases with recurrence or with high risk of recurrence, more choices of treatment are available in EMR than radiation, and EMR is able to be performed more safely than radiation. The complication rate after treatment is higher in surgery than other types of treatment. Subsequently, some patients were deceased within 1 month of treatment. In EMR and radiation, however, no treatment-related mortality has been noted and complication rates are almost equal, although the actual post-treatment complications are different between the two methods. Concerning quality of life after treatment, the esophagus is sacrificed during surgery, but EMR and radiation offer the benefit of postoperative preservation of the esophagus.

With regard to additional treatment after EMR/ESD, two cases in our series underwent radiotherapy. The patients had been pathologically diagnosed as being ly-positive following EMR and survived for long periods (118 and 34 months, respectively). Additional treatment for $\mathrm{m} 3$ and sm1 esophageal cancer following EMR is presently controversial. Such additional treatment would likely be minimally invasive and would enable patients to maintain their quality of life after treatment.
Accordingly, the previous form of treatment was surgery. However, if lymph node metastasis is not clearly visible on image examinations performed when additional treatment commences, radiotherapy or chemoradiotherapy may then be appropriate. However, intractable ulcers and mediastinitis can be lethal complications of radiotherapy and should not be overlooked (23). Long-term toxicity after chemoradiotherapy is also a serious complication (24). Therefore, to avoid unnecessary additional treatments, the use of additional treatments after EMR/ESD should be examined.

Follow-up examination with Lugol's staining and biopsies is performed every 3 months during the first year after EMR/ ESD and then every 6 months (25). Rigorous follow-up at regular intervals is necessary, due to the possibility of local recurrence. The rate of local recurrence after EMR has been reported as $15.4-22 \%$ (26-29). Numerous studies showed that piecemeal resection in particular would increase the risk of local recurrence $(1,26,30)$. ESD that has been performed in recent years allows for large en bloc resections, and is anticipated to be accepted as an important method for the expansion of the indications of endoscopic resection. Another study showed, however, that piecemeal resection did not increase the risk of local recurrence (27). Therefore, it is also necessary to investigate piecemeal resection by EMR/ESD in more detail. Although lymph node recurrence and distant metastasis of $m 3 \bullet s m 1$ cancer after EMR/ESD is a rare occurrence, follow-up at regular intervals with CT and other modalities is also necessary to detect early stage metastasis.

In conclusion, we suggest that lymphatic invasion is associated with lymph node metastasis in $\mathrm{m} 3$ or sm1 esophageal cancer. This association is a significant factor for treatment-related decision making. Our results suggest that patients diagnosed with $\mathrm{m} 3$ or $\mathrm{sm} 1$ esophageal cancer prior to treatment should undergo EMR/ESD as the first surgical strategy. If the resected tumor is diagnosed as ly positive, additional treatments for lymph node metastasis should then be considered. Furthermore, radiotherapy may be a choice for additional treatment, except for surgery. Strategies need to be developed to improve the diagnosis of depth of invasion, investigate predictors of lymph node metastasis and evaluate appropriate additional treatment after EMR/ESD. 


\section{References}

1. Oyama T, Tomori A, Hatta K, et al: Endoscopic submucosa dissection of early esophageal cancer. Clin Gastroenterol Hepatol 3: 67-70, 2005

2. Toyonaga $\mathrm{T}$, Nishino $\mathrm{E}$, Hirooka $\mathrm{T}$, et al: Use of short needle knife for esophageal endoscopic submucosal dissection. Dig Endosc 17: 246-252, 2005.

3. Eguchi T, Nakanishi Y, Shimoda T, et al: Histopathological criteria for additional treatment after endoscopic mucosal resection for esophageal cancer: analysis of 464 surgically resected cases. Mod Pathol 19: 475-480, 2006

4. Japan Esophageal Society: Japanese classification of esophageal cancer, 10th edition. Esophagus 6: 1-25, 2009.

5. Sugimachi K, Ikebe M, Kitamura K, Toh Y, Matsuda H and Kuwano H: Long-term results of esophagectomy for early esophageal carcinoma. Hepatogastroenterology 40: 203-206, 1993.

6. Tajima Y, Nakanishi Y, Ochiai A, et al: Histopathologic findings predicting lymph node metastasis and prognosis of patients with superficial esophageal carcinoma: analysis of 240 surgically resected tumors. Cancer 88: 1285-1293, 2000

7. Itoh Y, Fuwa N, Matsumoto A, Asano A and Sasaoka M: Treatment results of chemoradiation for T1 esophageal cancer with lymph node metastases. Radiat Med 18: 81-85, 2000.

8. Shimizu Y, Mera K, Tsukagoshi H, et al: Endoscopic ultrasonography for the detection of lymph node metastasis in superficial esophageal carcinoma. Dig Endosc 9: 178-182, 1997.

9. Kato H, Kuwano H, Nakajima M, et al: Comparison between positron emission tomography and computed tomography in the use of assessment of esophageal carcinoma. Cancer 94: 921-928, 2002.

10. Kato H, Miyazaki T, Nakajima M, et al: The incremental effect of positron emission tomography on diagnostic accuracy in the initial staging of esophageal carcinoma. Cancer 103: 148-156, 2005.

11. An JY, Baik YH, Noh JH, Sohn TS and Kim S: Predictive factors for lymph node metastasis in early gastric cancer with submucosal invasion: analysis of a single institutional experience. Ann Surg 246: 749-753, 2007.

12. Ueno $\mathrm{H}$, Mochizuki $\mathrm{H}$, Hashiguchi $\mathrm{Y}$, et al: Risk factor for an adverse outcome in early invasive colorectal carcinoma. Gastroenterology 127: 385-394, 2004.

13. Higuchi K, Tanabe S, Koizumi W, et al: Expansion of the indications for endoscopic mucosal resection in patients with superficial esophageal carcinoma. Endoscopy 39: 36-40, 2007.

14. Noguchi H, Naomoto Y, Kondo H, et al: Evaluation of endoscopic mucosal resection for superficial esophageal carcinoma. Surg Laparosc Endosc Percutan Thech 10: 343-350, 2000.

15. Makuuchi H, Shimada H, Mizutani K, et al: Endoscopic criteria for invasive depth of superficial esophageal cancer. Dig Endosc 9: $110-115,1997$.
16. May A, Gunter E, Roth F, et al: Accuracy of staging in early oesophageal cancer using high resolution endoscopy and high resolution endosonography: a comparative, prospective, and blinded trial. Gut 53: 634-640, 2004

17. Kato H, Momma K and Yoshida M: Early esophageal cancer: radiologic estimation of invasion into the muscularis mucosae. Abdom Imaging 28: 464-469, 2003.

18. Inoue $\mathrm{H}$, Honda $\mathrm{T}$, Yoshida $\mathrm{T}$, et al: Ultra-high magnification endoscopy of the normal esophageal mucosa. Dig Endosc 8: 134-138, 1996.

19. Inoue $H$, Honda $T$, Nagai $K$, et al: Ultra-high magnification endoscopic observation of carcinoma in situ of the esophagus. Dig Endosc 9: 16-18, 1997.

20. Katada C, Muto M, Momma K, et al: Clinical outcome after endoscopic mucosal resection for esophageal squamous cell carcinoma invading the muscularis mucosae - a multicenter retrospective cohort study. Endoscopy 39: 779-783, 2007.

21. Shimizu Y, Tsukagoshi H, Fujita M, Hosokawa M, Kato M and Asaka M: Long-term outcome after endoscopic mucosal resection in patients with esophageal squamous cell carcinoma invading muscularis mucosae or deeper. Gastrointest Endosc 56: 387-390, 2002.

22. Ishikawa H, Sakurai H, Tamaki Y, et al: Radiation therapy alone for stage I (UICC T1NOM0) squamous cell carcinoma of the esophagus: indication for surgery or combined chemoradiotherapy. J Gastroenterol Hepatol 21: 1290-1296, 2006.

23. Okawa T, Tanaka M, Kitaokawa M, et al: Superficial esophageal cancer: multi-center analysis of results of definitive radiation therapy in Japan. Radiology 196: 271-274, 1995.

24. Ishikura S, Nihei K, Ohtsu A, et al: Long-term toxicity after definitive chemoradiotherapy for squamous cell carcinoma of the thoracic esophagus. J Clin Oncol 21: 2697-2702, 2003.

25. Katada C, Muto M, Manabe T, Ohtsu A and Yoshida S: Local recurrence of squamous-cell carcinoma of the esophagus after EMR. Gastrointest Endosc 61: 219-225, 2005.

26. Ishihara R, Ishii H, Takeuchi Y, et al: Local recurrence of large squamous-cell carcinoma of the esophagus after endoscopic resection. Gastrointest Endosc 67: 799-804, 2008.

27. Kim JH, Chung HS, Youn YH, et al: Treatment outcomes of 70 cases of early esophageal carcinoma: 12 years of experience. Dis Esophagus 20: 297-300, 2007.

28. Esaki M, Matsumoto T, Hirakawa K, et al: Risk factors for local recurrence of superficial esophageal cancer after treatment by endoscopic mucosal resection. Endoscopy 39: 41-45, 2007.

29. Nomura T, Boku N, Ohtsu A, et al: Recurrence after endoscopic mucosal resection for superficial esophageal cancer. Endoscopy 32: 277-280, 2000.

30. Lin LF, Huang PT, Ho KS and Tung JN: Endoscopic mucosal resection of early esophageal carcinoma - experience of 9 cases. J Chin Med Assoc 71: 347-352, 2008. 